\section{Crystal structure of the solid solution $\left(\mathrm{Sr}_{1.65} \mathrm{~Pb}_{0.35}\right) \mathrm{Al}_{6} \mathrm{O}_{11}$}

\section{Matthias Weil}

Institute for Chemical Technologies and Analytics, Division of Structural Chemistry, Vienna University of Technology, Getreidemarkt 9/164-SC, A-1060 Vienna, Austria. *Correspondence e-mail: mweil@mail.zserv.tuwien.ac.at

Received 2 May 2014; accepted 6 May 2014

Edited by W. T. A. Harrison, University of Aberdeen, Scotland

The title compound, di(strontium/lead) hexaaluminate, is a member of the solid solution series $\left(\mathrm{Sr}_{2-x} \mathrm{~Pb}_{x}\right) \mathrm{Al}_{6} \mathrm{O}_{11}$. It contains two statistically occupied $M^{2+}(M=\mathrm{Sr}, \mathrm{Pb})$ sites [both with site symmetries ..m; Sr:Pb occupancy ratios = 0.756 (2):0.244 (2) and 0.8968 (19):0.1032 (19)] that are located in the voids of an aluminate framework. The $M^{2+}$ sites are surrounded by six and seven $\mathrm{O}$ atoms, respectively, if a cut-off $M-\mathrm{O}$ distance of $3 \AA$ is chosen, resulting in considerably distorted $M \mathrm{O}_{x}$ polyhedra. The aluminate framework consists of three $\mathrm{AlO}_{6}$ octahedra (two with point-group symmetries $.2 / m$ and one with ..2) sharing edges to form partially filled layers extending parallel to (100) and located at $x=0,0.5$. Adjacent $\mathrm{AlO}_{6}$ layers are linked by a network made up from two crystallographically different $\mathrm{AlO}_{4}$ tetrahedra by sharing corners.

Keywords: crystal structure; solid solution; aluminate framework.

CCDC reference: 1004262

\section{Related literature}

The title compound was obtained during experiments to prepare the strontium analogue of the lead calcium aluminate $\mathrm{PbCa}_{2} \mathrm{Al}_{8} \mathrm{O}_{15}$ (Artner \& Weil, 2012). For another member of the isotypic solid solution series $\left(\mathrm{Sr}_{2-x} \mathrm{~Pb}_{x}\right) \mathrm{Al}_{6} \mathrm{O}_{11}$ with a different Sr:Pb ratio, see: Plötz \& Müller-Buschbaum (1982).

\section{Experimental}

2.1. Crystal data

$\left(\mathrm{Sr}_{1.65} \mathrm{~Pb}_{0.35}\right) \mathrm{Al}_{6} \mathrm{O}_{11}$

$M_{r}=555.27$

Orthorhombic, Pnnm

$a=22.0299$ (4) $\AA$

$b=4.8802(1) \AA$

$c=8.3995$ (2) ̊

$V=903.03(3) \AA^{3}$

$Z=4$

Mo $K \alpha$ radiation

$\mu=16.94 \mathrm{~mm}^{-1}$

$T=296 \mathrm{~K}$

$0.12 \times 0.05 \times 0.05 \mathrm{~mm}$

\subsection{Data collection}

Bruker SMART CCD

diffractometer

Absorption correction: multi-scan

(SADABS; Bruker, 2008)

$T_{\min }=0.236, T_{\max }=0.485$

10434 measured reflections 2509 independent reflections 2190 reflections with $I>2 \sigma(I)$

$R_{\text {int }}=0.042$

\subsection{Refinement}

$R\left[F^{2}>2 \sigma\left(F^{2}\right)\right]=0.027$

$w R\left(F^{2}\right)=0.061$

$S=1.05$

2509 reflections

$$
\begin{aligned}
& 101 \text { parameters } \\
& \Delta \rho_{\max }=1.12 \mathrm{e} \AA^{-3} \\
& \Delta \rho_{\min }=-1.51 \mathrm{e}^{-3}
\end{aligned}
$$

Data collection: SMART (Bruker, 2008); cell refinement: SAINT (Bruker, 2008); data reduction: $S A I N T$; $\operatorname{program}(\mathrm{s})$ used to solve structure: SHELXS97 (Sheldrick, 2008); program(s) used to refine structure: SHELXL97 (Sheldrick, 2008); molecular graphics: ATOMS for Windows (Dowty, 2006); software used to prepare material for publication: publCIF (Westrip, 2010).

\section{Acknowledgements}

The X-ray centre of the Vienna University of Technology is acknowledged for providing access to the single-crystal diffractometer.

Supporting information for this paper is available from the IUCr electronic archives (Reference: HB0009).

\section{References}

Artner, C. \& Weil, M. (2012). Acta Cryst. C68, i1-i3.

Bruker (2008). SMART, SAINT and SADABS. Bruker AXS Inc. Madison, Wisconsin, USA.

Dowty, E. (2006). ATOMS for Windows. Shape Software, Kingsport, Tennessee, USA.

Plötz, K. B. \& Müller-Buschbaum, H. (1982). Z. Anorg. Allg. Chem. 491, $253-$ 258.

Sheldrick, G. M. (2008). Acta Cryst. A64, 112-122.

Westrip, S. P. (2010). J. Appl. Cryst. 43, 920-925. 


\section{supporting information}

Acta Cryst. (2014). E70, i45 [doi:10.1107/S1600536814010216]

\section{Crystal structure of the solid solution $\left(\mathrm{Sr}_{1.65} \mathrm{~Pb}_{0.35}\right) \mathrm{Al}_{6} \mathrm{O}_{11}$}

\section{Matthias Weil}

\section{S1. Experimental}

A mixture of $2 \mathrm{PbCO}_{3} \cdot \mathrm{Pb}(\mathrm{OH})_{2}, \mathrm{SrCO}_{3}$ and $\mathrm{Al}(\mathrm{OH})_{3}$ (molar ratio 1:6:24) was heated in a platinum crucible over a period of $24 \mathrm{~h}$ to $1233 \mathrm{~K}$, kept at this temperature for $10 \mathrm{~h}$ and cooled over a period of $24 \mathrm{~h}$ to room temperature. A few colourless platy crystals of the title compound grew on top of a brick-red microcrystalline matrix that was not further analysed.

\section{S2. Refinement}

Atom labels and starting parameters for refinement were taken from the isotypic compound $\left(\mathrm{Sr}_{1.33} \mathrm{~Pb}_{0.67}\right) \mathrm{Al}_{6} \mathrm{O}_{11}(\mathrm{Plötz} \mathrm{\&}$ Müller-Buschbaum, 1982). The two $M^{2+}$ sites are statistically occupied by $\mathrm{Pb}$ and $\mathrm{Sr}$. For each site, full occupancy was considered and the occupancy factors refined independently. The highest and lowest remaining electron densities are located $0.64 \AA$ and $0.72 \AA$, respectively, from the site $(\mathrm{Sr} 1 / \mathrm{Pb} 1)$. 


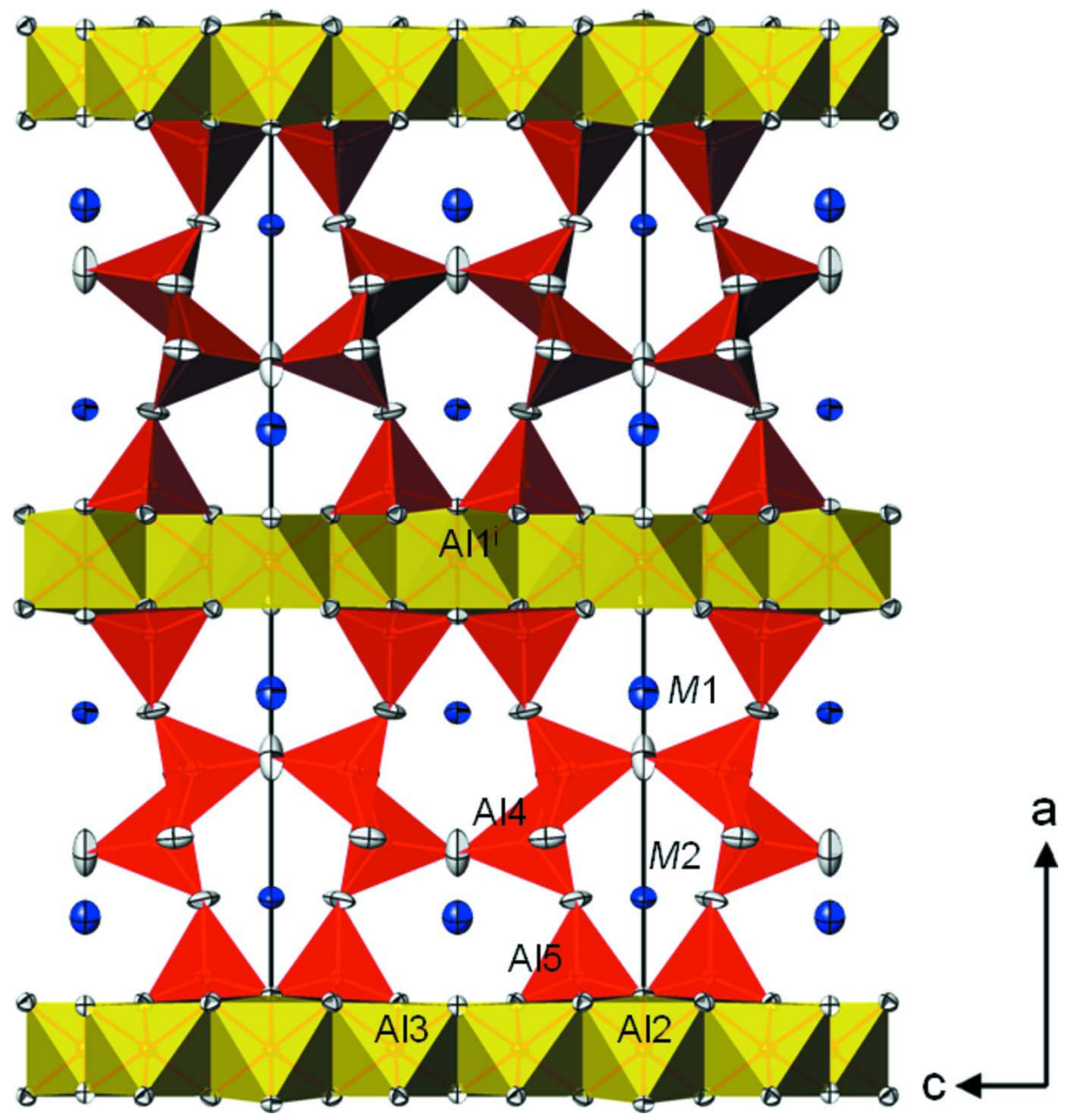

\section{Figure 1}

The crystal structure of $\left(\mathrm{Sr}_{1.65} \mathrm{~Pb}_{0.35}\right) \mathrm{Al}_{6} \mathrm{O}_{11}$ in a projection along [010]. $\mathrm{AlO}_{6}$ octahedra are yellow, $\mathrm{AlO}_{4}$ octahedra are red. Displacement ellipsoids are drawn at the $97 \%$ probability level. Bonds to the statistically occupied $M^{2+}$ sites $(M=\mathrm{Sr}, \mathrm{Pb})$ were omitted for clarity. [Symmetry code: i) $x+1 / 2, y, z$.] 


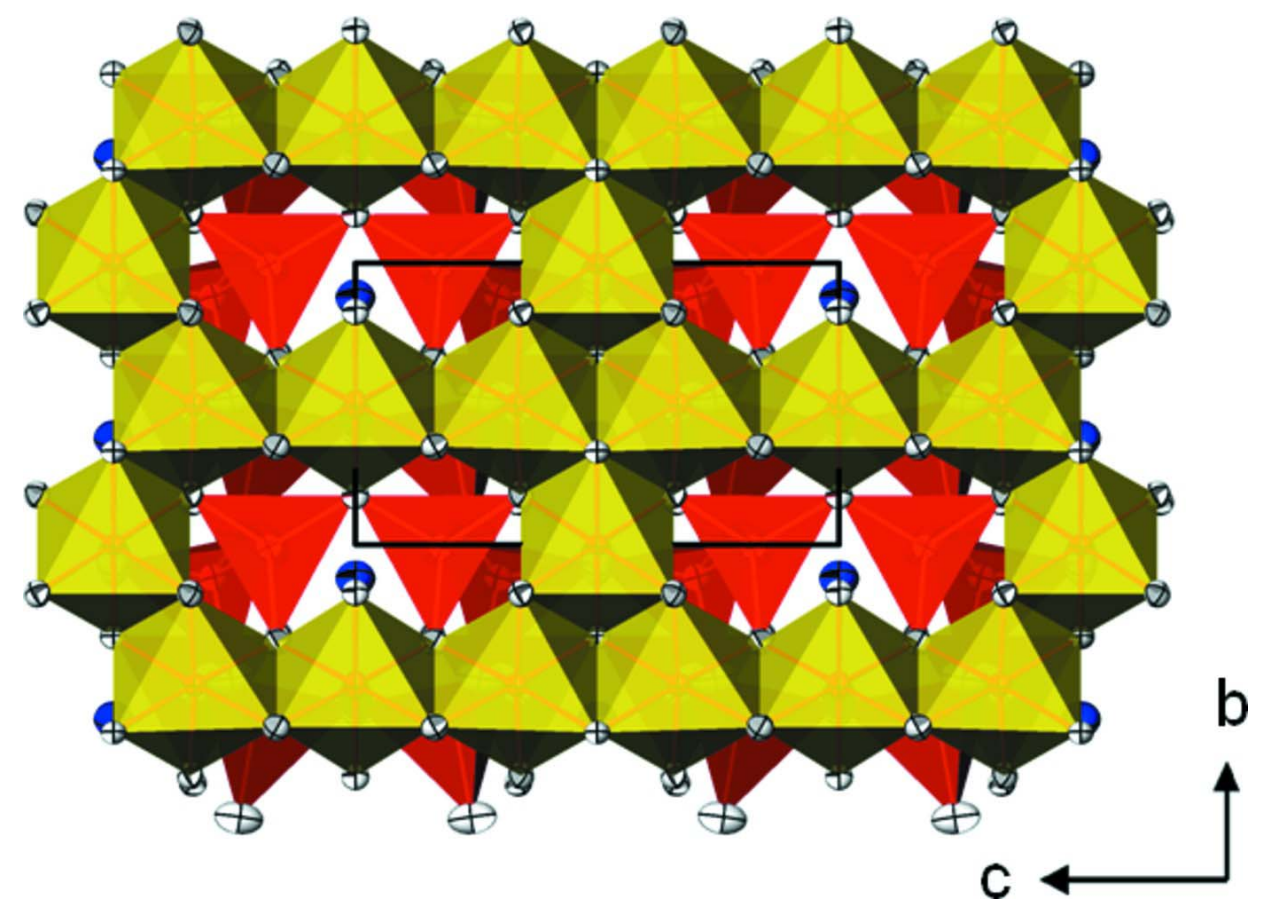

\section{Figure 2}

The crystal structure of $\left(\mathrm{Sr}_{1.65} \mathrm{~Pb}_{0.35}\right) \mathrm{Al}_{6} \mathrm{O}_{11}$ in a projection along [100] showing one layer of edge-sharing $\mathrm{AlO}_{6}$ octahedra and the connecting layer of corner-sharing $\mathrm{AlO}_{4}$ tetrahedra as well as one layer of $M^{2+}$ sites $(M=\mathrm{Sr}, \mathrm{Pb})$. Colour code and displacement ellipsoids are as in Fig. 1.

\section{Di(strontium/lead) hexaaluminate}

\section{Crystal data}

$\left(\mathrm{Sr}_{1.65} \mathrm{~Pb}_{0.35}\right) \mathrm{Al}_{6} \mathrm{O}_{11}$

$M_{r}=555.27$

Orthorhombic, Pnnm

Hall symbol: -P 2 2n

$a=22.0299(4) \AA$

$b=4.8802(1) \AA$

$c=8.3995(2) \AA$

$V=903.03(3) \AA^{3}$

$Z=4$

\section{Data collection}

\section{Bruker SMART CCD \\ diffractometer}

Radiation source: fine-focus sealed tube

Graphite monochromator

$\omega$ scans

Absorption correction: multi-scan

(SADABS; Bruker, 2008)

$T_{\min }=0.236, T_{\max }=0.485$
$F(000)=1030$

$D_{\mathrm{x}}=4.084 \mathrm{Mg} \mathrm{m}^{-3}$

Mo $K \alpha$ radiation, $\lambda=0.71073 \AA$

Cell parameters from 3598 reflections

$\theta=3.7-32.5^{\circ}$

$\mu=16.94 \mathrm{~mm}^{-1}$

$T=296 \mathrm{~K}$

Plate, colourless

$0.12 \times 0.05 \times 0.05 \mathrm{~mm}$

10434 measured reflections

2509 independent reflections

2190 reflections with $I>2 \sigma(I)$

$R_{\text {int }}=0.042$

$\theta_{\text {max }}=37.5^{\circ}, \theta_{\text {min }}=3.7^{\circ}$

$h=-30 \rightarrow 37$

$k=-6 \rightarrow 8$

$l=-14 \rightarrow 9$ 


\section{Refinement}

Refinement on $F^{2}$

Least-squares matrix: full

$R\left[F^{2}>2 \sigma\left(F^{2}\right)\right]=0.027$

$w R\left(F^{2}\right)=0.061$

$S=1.05$

2509 reflections

101 parameters

0 restraints
Primary atom site location: isomorphous structure methods

$w=1 /\left[\sigma^{2}\left(F_{\mathrm{o}}^{2}\right)+(0.0244 P)^{2}+0.7036 P\right]$

where $P=\left(F_{\mathrm{o}}{ }^{2}+2 F_{\mathrm{c}}{ }^{2}\right) / 3$

$(\Delta / \sigma)_{\max }=0.001$

$\Delta \rho_{\max }=1.12 \mathrm{e} \AA^{-3}$

$\Delta \rho_{\min }=-1.51$ e $\AA^{-3}$

Extinction correction: SHELXL97 (Sheldrick, 2008), $\mathrm{Fc}^{*}=\mathrm{kFc}\left[1+0.001 \mathrm{xFc}^{2} \lambda^{3} / \sin (2 \theta)\right]^{-1 / 4}$

Extinction coefficient: 0.0022 (3)

\section{Special details}

Geometry. All e.s.d.'s (except the e.s.d. in the dihedral angle between two 1.s. planes) are estimated using the full covariance matrix. The cell e.s.d.'s are taken into account individually in the estimation of e.s.d.'s in distances, angles and torsion angles; correlations between e.s.d.'s in cell parameters are only used when they are defined by crystal symmetry. An approximate (isotropic) treatment of cell e.s.d.'s is used for estimating e.s.d.'s involving 1.s. planes.

Refinement. Refinement of $F^{2}$ against ALL reflections. The weighted $R$-factor $w R$ and goodness of fit $S$ are based on $F^{2}$, conventional $R$-factors $R$ are based on $F$, with $F$ set to zero for negative $F^{2}$. The threshold expression of $F^{2}>\sigma\left(F^{2}\right)$ is used only for calculating $R$-factors (gt) etc. and is not relevant to the choice of reflections for refinement. $R$-factors based on $F^{2}$ are statistically about twice as large as those based on $F$, and $R$ - factors based on ALL data will be even larger.

Fractional atomic coordinates and isotropic or equivalent isotropic displacement parameters $\left(\AA^{2}\right)$

\begin{tabular}{llllll}
\hline & $x$ & $y$ & $z$ & $U_{\text {iso }} * / U_{\text {eq }}$ & Occ. $(<1)$ \\
\hline Pb1 & $0.364795(11)$ & $0.12254(4)$ & 0.0000 & $0.01369(7)$ & $0.756(2)$ \\
$\mathrm{Pb} 2$ & $0.155369(11)$ & $0.54620(5)$ & 0.0000 & $0.01112(8)$ & $0.8968(19)$ \\
$\mathrm{Sr} 1$ & $0.364795(11)$ & $0.12254(4)$ & 0.0000 & $0.01369(7)$ & $0.244(2)$ \\
$\mathrm{S} 2$ & $0.155369(11)$ & $0.54620(5)$ & 0.0000 & $0.01112(8)$ & $0.1032(19)$ \\
$\mathrm{A} 11$ & 0.0000 & 0.0000 & 0.5000 & $0.00496(19)$ & \\
$\mathrm{A} 12$ & 0.0000 & 0.5000 & 0.0000 & $0.00509(19)$ & \\
$\mathrm{A} 13$ & 0.0000 & 0.5000 & $0.66773(9)$ & $0.00472(14)$ & \\
$\mathrm{A} 14$ & $0.21348(3)$ & $0.11886(11)$ & $0.29528(7)$ & $0.00638(12)$ & \\
$\mathrm{A} 15$ & $0.07460(3)$ & $0.00878(11)$ & $0.17903(6)$ & $0.00472(11)$ & \\
O1 & $0.05605(9)$ & $0.8271(4)$ & 0.0000 & $0.0055(3)$ & \\
O2 & $0.29866(14)$ & $0.5677(5)$ & 0.0000 & $0.0197(5)$ & \\
O3 & $0.45181(6)$ & $0.3230(3)$ & $0.84219(15)$ & $0.0060(2)$ & \\
O4 & $0.34669(7)$ & $0.4960(3)$ & $0.31766(19)$ & $0.0118(3)$ & \\
O5 & $0.45552(9)$ & $0.8297(4)$ & 0.0000 & $0.0055(3)$ & \\
O6 & $0.95192(6)$ & $0.6515(3)$ & $0.83661(15)$ & $0.0062(2)$ & \\
O7 & $0.28328(7)$ & $0.9728(3)$ & $0.24121(19)$ & $0.0117(3)$ & \\
& & & & & \\
\hline
\end{tabular}

Atomic displacement parameters $\left(\AA^{2}\right)$

\begin{tabular}{lllllll}
\hline & $U^{11}$ & $U^{22}$ & $U^{33}$ & $U^{12}$ & $U^{13}$ & $U^{23}$ \\
\hline $\mathrm{Pb} 1$ & $0.01691(12)$ & $0.01109(9)$ & $0.01306(9)$ & $0.00260(7)$ & 0.000 & 0.000 \\
$\mathrm{~Pb} 2$ & $0.00748(12)$ & $0.01603(12)$ & $0.00985(10)$ & $0.00238(8)$ & 0.000 & 0.000 \\
$\mathrm{Sr} 1$ & $0.01691(12)$ & $0.01109(9)$ & $0.01306(9)$ & $0.00260(7)$ & 0.000 & 0.000 \\
$\mathrm{Sr} 2$ & $0.00748(12)$ & $0.01603(12)$ & $0.00985(10)$ & $0.00238(8)$ & 0.000 & 0.000 \\
$\mathrm{~A} 11$ & $0.0059(5)$ & $0.0041(4)$ & $0.0049(4)$ & $-0.0004(4)$ & 0.000 & 0.000
\end{tabular}


supporting information

\begin{tabular}{lllllll}
$\mathrm{A} 12$ & $0.0039(5)$ & $0.0070(4)$ & $0.0044(4)$ & $0.0012(4)$ & 0.000 & 0.000 \\
$\mathrm{~A} 13$ & $0.0043(3)$ & $0.0048(3)$ & $0.0050(3)$ & $0.0001(2)$ & 0.000 & 0.000 \\
$\mathrm{~A} 14$ & $0.0049(3)$ & $0.0067(2)$ & $0.0075(2)$ & $-0.00021(18)$ & $-0.00054(18)$ & $0.00025(16)$ \\
$\mathrm{A} 15$ & $0.0035(2)$ & $0.0052(2)$ & $0.0054(2)$ & $-0.00008(18)$ & $0.00009(17)$ & $0.00014(16)$ \\
$\mathrm{O} 1$ & $0.0041(8)$ & $0.0060(7)$ & $0.0063(7)$ & $-0.0011(6)$ & 0.000 & 0.000 \\
$\mathrm{O} 2$ & $0.0319(15)$ & $0.0187(10)$ & $0.0086(8)$ & $0.0063(10)$ & 0.000 & 0.000 \\
$\mathrm{O} 3$ & $0.0058(6)$ & $0.0064(5)$ & $0.0058(5)$ & $0.0007(4)$ & $-0.0006(4)$ & $0.0001(4)$ \\
$\mathrm{O} 4$ & $0.0046(6)$ & $0.0151(6)$ & $0.0157(6)$ & $-0.0009(5)$ & $-0.0023(5)$ & $0.0065(5)$ \\
$\mathrm{O} 5$ & $0.0065(8)$ & $0.0045(7)$ & $0.0055(6)$ & $-0.0002(6)$ & 0.000 & 0.000 \\
$\mathrm{O} 6$ & $0.0058(6)$ & $0.0063(5)$ & $0.0065(5)$ & $0.0013(4)$ & $-0.0007(4)$ & $0.0000(4)$ \\
O7 & $0.0067(6)$ & $0.0087(6)$ & $0.0196(7)$ & $0.0000(5)$ & $-0.0011(5)$ & $-0.0016(5)$ \\
\hline
\end{tabular}

Geometric parameters $\left(\AA,{ }^{\circ}\right)$

\begin{tabular}{|c|c|c|c|}
\hline $\mathrm{Pb} 1-\mathrm{O}^{\mathrm{i}}$ & $2.4569(19)$ & $\mathrm{Al} 1-\mathrm{O} 3^{\mathrm{vii}}$ & $1.9055(13)$ \\
\hline $\mathrm{Pb} 1-\mathrm{O}^{\mathrm{ii}}$ & $2.5275(13)$ & $\mathrm{A} 11-\mathrm{O}^{\mathrm{xv}}$ & $1.9055(13)$ \\
\hline $\mathrm{Pb} 1-\mathrm{O} 3^{\mathrm{iii}}$ & $2.5275(13)$ & $\mathrm{A} 12-\mathrm{O}^{\mathrm{xi}}$ & $1.8847(13)$ \\
\hline $\mathrm{Pb} 1-\mathrm{O} 2$ & $2.616(3)$ & $\mathrm{A} 12-\mathrm{O}^{\mathrm{xvi}}$ & $1.8847(13)$ \\
\hline $\mathrm{Pb} 1-\mathrm{O}^{\text {iv }}$ & $2.8042(16)$ & $\mathrm{A} 12-\mathrm{O}^{\mathrm{xvii}}$ & $1.8847(13)$ \\
\hline $\mathrm{Pb} 1-\mathrm{O}^{\mathrm{i}}$ & $2.8042(16)$ & $\mathrm{Al} 2-\mathrm{O}^{\mathrm{x}}$ & $1.8847(13)$ \\
\hline $\mathrm{Pb} 1-\mathrm{O} 2^{\mathrm{i}}$ & $3.075(3)$ & $\mathrm{A} 12-\mathrm{O} 1$ & $2.0181(19)$ \\
\hline $\mathrm{Pb} 1-\mathrm{O} 4$ & $3.2558(17)$ & $\mathrm{A} 12-\mathrm{O} 1^{\text {xviii }}$ & $2.0181(19)$ \\
\hline $\mathrm{Pb} 1-\mathrm{O} 4^{\mathrm{v}}$ & $3.2558(17)$ & $\mathrm{Al} 3-\mathrm{O} 3^{\mathrm{xv}}$ & $1.9021(13)$ \\
\hline $\mathrm{Pb} 2-\mathrm{O} 1$ & $2.582(2)$ & $\mathrm{Al} 3-\mathrm{O}^{\mathrm{xix}}$ & $1.9021(13)$ \\
\hline $\mathrm{Pb} 2-\mathrm{O} 7^{\mathrm{vi}}$ & $2.5846(16)$ & $\mathrm{Al} 3-\mathrm{O} 5^{\mathrm{xx}}$ & $1.9067(14)$ \\
\hline $\mathrm{Pb} 2-\mathrm{O} 7^{\mathrm{vii}}$ & $2.5846(16)$ & $\mathrm{Al} 3-\mathrm{O}^{\mathrm{vi}}$ & 1.9067 (14) \\
\hline $\mathrm{Pb} 2-\mathrm{O} 4^{\text {viii }}$ & $2.6771(15)$ & $\mathrm{Al} 3-\mathrm{O} 6^{\mathrm{xxi}}$ & $1.9186(14)$ \\
\hline $\mathrm{Pb} 2-\mathrm{O} 4^{\mathrm{ix}}$ & $2.6771(15)$ & $\mathrm{Al} 3-\mathrm{O}^{\mathrm{xxii}}$ & $1.9186(14)$ \\
\hline $\mathrm{Pb} 2-\mathrm{O}^{\mathrm{x}}$ & $2.8983(14)$ & $\mathrm{Al} 4-\mathrm{O} 4^{\mathrm{vi}}$ & $1.7368(16)$ \\
\hline $\mathrm{Pb} 2-\mathrm{O}^{\mathrm{xi}}$ & $2.8983(14)$ & $\mathrm{Al} 4-\mathrm{O}^{\mathrm{i}}$ & $1.7548(17)$ \\
\hline $\mathrm{Pb} 2-\mathrm{O} 4^{\text {vii }}$ & $3.0914(17)$ & $\mathrm{Al} 4-\mathrm{O}^{\mathrm{vi}}$ & $1.7556(16)$ \\
\hline $\mathrm{Pb} 2-\mathrm{O} 4^{\mathrm{vi}}$ & $3.0914(17)$ & $\mathrm{A} 14-\mathrm{O} 2^{\mathrm{vi}}$ & $1.7581(8)$ \\
\hline $\mathrm{Pb} 2-\mathrm{O} 2$ & $3.158(3)$ & $\mathrm{A} 15-\mathrm{O} 4^{\mathrm{vi}}$ & $1.7352(16)$ \\
\hline $\mathrm{Al} 1-\mathrm{O} 5^{\mathrm{xii}}$ & $1.8841(18)$ & $\mathrm{A} 15-\mathrm{O} 3^{\mathrm{vii}}$ & $1.7433(14)$ \\
\hline $\mathrm{A} 11-\mathrm{O}^{\mathrm{vi}}$ & $1.8841(18)$ & $\mathrm{A} 15-\mathrm{O}^{\mathrm{xi}}$ & $1.7627(14)$ \\
\hline $\mathrm{A} 11-\mathrm{O} 3^{\mathrm{xiii}}$ & 1.9055 (13) & $\mathrm{A} 15-\mathrm{O} 1^{\mathrm{i}}$ & $1.7929(11)$ \\
\hline $\mathrm{A} 11-\mathrm{O}^{\mathrm{xiv}}$ & 1.9055 (13) & & \\
\hline $\mathrm{O} 5^{\mathrm{i}}-\mathrm{Pb} 1-\mathrm{O} 3^{\mathrm{ii}}$ & $66.93(5)$ & $\mathrm{O} 6^{\mathrm{xvi}}-\mathrm{A} 12-\mathrm{O} 6^{\mathrm{x}}$ & 180.0 \\
\hline $\mathrm{O} 5^{\mathrm{i}}-\mathrm{Pb} 1-\mathrm{O} 3^{\mathrm{iii}}$ & $66.93(5)$ & $\mathrm{O} 6^{\mathrm{xi}}-\mathrm{A} 12-\mathrm{O} 1$ & $88.08(5)$ \\
\hline 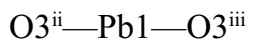 & $63.26(6)$ & $\mathrm{O} 6^{\mathrm{xvi}}-\mathrm{A} 12-\mathrm{O} 1$ & $91.92(5)$ \\
\hline $\mathrm{O} 5^{\mathrm{i}}-\mathrm{Pb} 1-\mathrm{O} 2$ & $159.41(8)$ & $\mathrm{O} 6^{\mathrm{xvi}}-\mathrm{A} 12-\mathrm{O} 1$ & $91.92(5)$ \\
\hline $\mathrm{O} 3^{\mathrm{ii}-\mathrm{Pb} 1-\mathrm{O} 2}$ & $95.80(6)$ & $\mathrm{O}^{\mathrm{x}}-\mathrm{A} 12-\mathrm{O} 1$ & $88.08(5)$ \\
\hline $\mathrm{O} 3^{\mathrm{iii}-\mathrm{Pb} 1-\mathrm{O} 2}$ & $95.80(6)$ & $\mathrm{O} 6^{\mathrm{xi}}-\mathrm{A} 12-\mathrm{O} 1^{\mathrm{xviii}}$ & $91.92(5)$ \\
\hline $\mathrm{O} 5^{\mathrm{i}}-\mathrm{Pb} 1-\mathrm{O} 7^{\mathrm{iv}}$ & $111.67(4)$ & $\mathrm{O} 6^{\mathrm{xvi}}-\mathrm{A} 12-\mathrm{O} 1^{\mathrm{xviii}}$ & $88.08(5)$ \\
\hline $\mathrm{O} 3^{\mathrm{ii}}-\mathrm{Pb} 1-\mathrm{O} 7^{\mathrm{iv}}$ & $164.89(4)$ & $\mathrm{O} 6^{\mathrm{xvii}}-\mathrm{A} 12-\mathrm{O} 1^{\mathrm{xviii}}$ & $88.08(5)$ \\
\hline 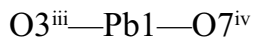 & $101.98(4)$ & $\mathrm{O} 6^{\mathrm{x}}-\mathrm{A} 12-\mathrm{O} 1^{\mathrm{xviii}}$ & $91.92(5)$ \\
\hline $\mathrm{O} 2-\mathrm{Pb} 1-\mathrm{O} 7^{\mathrm{iv}}$ & $81.94(5)$ & $\mathrm{O} 1-\mathrm{A} 12-\mathrm{O}^{\text {xviii }}$ & 180.0 \\
\hline
\end{tabular}




\begin{tabular}{|c|c|}
\hline $\mathrm{O} 5^{\mathrm{i}}-\mathrm{Pb} 1-\mathrm{O}^{\mathrm{i}}$ & $111.67(4)$ \\
\hline $\mathrm{O} 3^{\mathrm{ii}}-\mathrm{Pb} 1-\mathrm{O} 7^{\mathrm{i}}$ & $101.98(4)$ \\
\hline 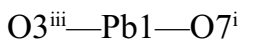 & $164.89(4)$ \\
\hline $\mathrm{O} 2-\mathrm{Pb} 1-\mathrm{O} 7^{\mathrm{i}}$ & $81.94(5)$ \\
\hline $\mathrm{O}^{\mathrm{iv}}-\mathrm{Pb} 1-\mathrm{O}^{\mathrm{i}}$ & $92.52(6)$ \\
\hline $\mathrm{O} 5^{\mathrm{i}}-\mathrm{Pb} 1-\mathrm{O} 2^{\mathrm{i}}$ & $82.72(7)$ \\
\hline $\mathrm{O} 3^{\mathrm{ii}}-\mathrm{Pb} 1-\mathrm{O} 2^{\mathrm{i}}$ & $134.44(5)$ \\
\hline 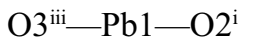 & $134.44(5)$ \\
\hline $\mathrm{O} 2-\mathrm{Pb} 1-\mathrm{O} 2^{\mathrm{i}}$ & $117.87(11)$ \\
\hline $\mathrm{O} 7^{\mathrm{iv}}-\mathrm{Pb} 1-\mathrm{O} 2^{\mathrm{i}}$ & $57.79(4)$ \\
\hline $\mathrm{O} 7^{\mathrm{i}}-\mathrm{Pb} 1-\mathrm{O} 2^{\mathrm{i}}$ & $57.79(4)$ \\
\hline $\mathrm{O} 5$ i- $\mathrm{Pb} 1-\mathrm{O} 4$ & $115.16(3)$ \\
\hline $\mathrm{O} 33^{\mathrm{ii}}-\mathrm{Pb} 1-\mathrm{O} 4$ & $56.39(4)$ \\
\hline $\mathrm{O} 3{ }^{\mathrm{iii}}-\mathrm{Pb} 1-\mathrm{O} 4$ & $107.82(4)$ \\
\hline $\mathrm{O} 2-\mathrm{Pb} 1-\mathrm{O} 4$ & $57.78(3)$ \\
\hline $\mathrm{O}^{\mathrm{iv}}-\mathrm{Pb} 1-\mathrm{O} 4$ & $131.27(4)$ \\
\hline $\mathrm{O} 7-\mathrm{Pb} 1-\mathrm{O} 4$ & $58.36(4)$ \\
\hline $\mathrm{O} 22^{\mathrm{i}}-\mathrm{Pb} 1-\mathrm{O} 4$ & $115.78(3)$ \\
\hline $\mathrm{O} 5^{\mathrm{i}}-\mathrm{Pb} 1-\mathrm{O}^{\mathrm{v}}$ & $115.16(3)$ \\
\hline 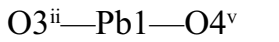 & $107.82(4)$ \\
\hline 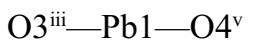 & $56.39(4)$ \\
\hline $\mathrm{O} 2-\mathrm{Pb} 1-\mathrm{O} 4^{\mathrm{v}}$ & $57.78(3)$ \\
\hline $\mathrm{O}^{\mathrm{iv}}-\mathrm{Pb} 1-\mathrm{O}^{\mathrm{v}}$ & $58.36(4)$ \\
\hline $\mathrm{O} 7^{\mathrm{i}}-\mathrm{Pb} 1-\mathrm{O} 4^{\mathrm{v}}$ & $131.27(4)$ \\
\hline $\mathrm{O} 2^{\mathrm{i}}-\mathrm{Pb} 1-\mathrm{O} 4^{\mathrm{v}}$ & $115.78(3)$ \\
\hline $\mathrm{O} 4-\mathrm{Pb} 1-\mathrm{O}^{\mathrm{v}}$ & $110.07(5)$ \\
\hline $\mathrm{O} 1-\mathrm{Pb} 2-\mathrm{O} 7^{\mathrm{vi}}$ & $121.11(4)$ \\
\hline $\mathrm{O} 1-\mathrm{Pb} 2-\mathrm{O} 7^{\mathrm{vii}}$ & $121.11(4)$ \\
\hline $\mathrm{O} 7^{\mathrm{vi}}-\mathrm{Pb} 2-\mathrm{O} 7^{\mathrm{vii}}$ & $114.50(7)$ \\
\hline $\mathrm{O} 1-\mathrm{Pb} 2-\mathrm{O} 4^{\text {viii }}$ & $63.27(5)$ \\
\hline $\mathrm{O} 7^{\mathrm{vi}}-\mathrm{Pb} 2-\mathrm{O} 4^{\mathrm{viii}}$ & $68.99(5)$ \\
\hline $\mathrm{O} 7^{\mathrm{vii}}-\mathrm{Pb} 2-\mathrm{O} 4^{\mathrm{viii}}$ & $127.14(5)$ \\
\hline $\mathrm{O} 1-\mathrm{Pb} 2-\mathrm{O} 4^{\mathrm{ix}}$ & $63.27(4)$ \\
\hline $\mathrm{O} 7^{\mathrm{vi}}-\mathrm{Pb} 2-\mathrm{O}^{\mathrm{ix}}$ & $127.14(5)$ \\
\hline 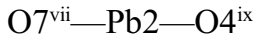 & $68.99(5)$ \\
\hline $\mathrm{O} 4^{\mathrm{viii}}-\mathrm{Pb} 2-\mathrm{O} 4^{\mathrm{ix}}$ & $69.79(7)$ \\
\hline $\mathrm{O} 1-\mathrm{Pb} 2-\mathrm{O}^{\mathrm{x}}$ & $59.05(4)$ \\
\hline $\mathrm{O} 7^{\mathrm{vi}}-\mathrm{Pb} 2-\mathrm{O}^{\mathrm{x}}$ & $141.12(4)$ \\
\hline $\mathrm{O} 7^{\mathrm{vii}}-\mathrm{Pb} 2-\mathrm{O}^{\mathrm{x}}$ & $88.97(4)$ \\
\hline $\mathrm{O} 4^{\mathrm{vii}}-\mathrm{Pb} 2-\mathrm{O}^{\mathrm{x}}$ & $122.01(4)$ \\
\hline $\mathrm{O} 4^{\mathrm{ix}}-\mathrm{Pb} 2-\mathrm{O}^{\mathrm{x}}$ & $89.33(4)$ \\
\hline $\mathrm{O} 1-\mathrm{Pb} 2-\mathrm{O}^{\mathrm{xi}}$ & $59.05(4)$ \\
\hline $\mathrm{O} 7^{\mathrm{vi}}-\mathrm{Pb} 2-\mathrm{O}^{\mathrm{xi}}$ & $88.97(4)$ \\
\hline $\mathrm{O} 7^{\mathrm{vii}}-\mathrm{Pb} 2-\mathrm{O}^{\mathrm{xi}}$ & $141.12(4)$ \\
\hline $\mathrm{O} 4^{\mathrm{vii}}-\mathrm{Pb} 2-\mathrm{O}^{\mathrm{xi}}$ & $89.33(4)$ \\
\hline $\mathrm{O} 4^{\mathrm{ix}}-\mathrm{Pb} 2-\mathrm{O}^{\mathrm{xi}}$ & $122.01(4)$ \\
\hline $\mathrm{O} 6^{\mathrm{x}}-\mathrm{Pb} 2-\mathrm{O}^{\mathrm{xi}}$ & $56.52(5)$ \\
\hline $\mathrm{O} 1-\mathrm{Pb} 2-\mathrm{O} 4^{\text {vii }}$ & $116.66(5)$ \\
\hline
\end{tabular}

\begin{tabular}{|c|c|}
\hline $\mathrm{O} 3^{\mathrm{xv}}-\mathrm{A} 13-\mathrm{O} 3^{\mathrm{xix}}$ & $174.98(9)$ \\
\hline $\mathrm{O} 3^{\mathrm{xy}}-\mathrm{A} 13-\mathrm{O} 5^{\mathrm{xx}}$ & $92.40(7)$ \\
\hline $\mathrm{O} 3^{\mathrm{xix}}-\mathrm{A} 13-\mathrm{O} 5^{\mathrm{xx}}$ & $83.88(7)$ \\
\hline $\mathrm{O}^{\mathrm{xv}}-\mathrm{A} 13-\mathrm{O}^{\mathrm{vi}}$ & $83.88(7)$ \\
\hline $\mathrm{O} 3^{\mathrm{xix}}-\mathrm{A} 13-\mathrm{O} 5^{\mathrm{vi}}$ & $92.40(7)$ \\
\hline $\mathrm{O}^{\mathrm{xx}}-\mathrm{A} 13-\mathrm{O}^{\mathrm{vi}}$ & $84.72(9)$ \\
\hline $\mathrm{O} 3^{\mathrm{xv}}-\mathrm{A} 13-\mathrm{O}^{\mathrm{xxi}}$ & $91.22(6)$ \\
\hline $\mathrm{O} 3^{\mathrm{xix}}-\mathrm{A} 13-\mathrm{O}^{\mathrm{xxi}}$ & $92.49(6)$ \\
\hline $\mathrm{O} 5^{\mathrm{xx}}-\mathrm{A} 13-\mathrm{O} 6^{\mathrm{xxi}}$ & $176.38(7)$ \\
\hline $\mathrm{O} 5^{\mathrm{vi}}-\mathrm{Al} 3-\mathrm{O}^{\mathrm{xxi}}$ & $95.43(6)$ \\
\hline $\mathrm{O} 3^{\mathrm{xv}}-\mathrm{A} 13-\mathrm{O}^{\mathrm{xxii}}$ & $92.49(6)$ \\
\hline $\mathrm{O} 3^{\mathrm{xix}}-\mathrm{Al} 3-\mathrm{O} 6^{\mathrm{xxii}}$ & $91.22(6)$ \\
\hline $\mathrm{O} 5^{\mathrm{xx}}-\mathrm{A} 13-\mathrm{O} 6^{\mathrm{xxii}}$ & $95.43(6)$ \\
\hline $\mathrm{O} 5^{\mathrm{vi}}-\mathrm{A} 13-\mathrm{O}^{\mathrm{xxii}}$ & $176.38(7)$ \\
\hline $\mathrm{O} 6^{\mathrm{xxi}}-\mathrm{A} 13-\mathrm{O} 6^{\mathrm{xxii}}$ & $84.65(9)$ \\
\hline 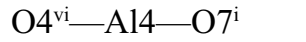 & $112.79(8)$ \\
\hline $\mathrm{O} 4^{\mathrm{vi}}-\mathrm{A} 14-\mathrm{O} 7^{\mathrm{vi}}$ & $105.98(8)$ \\
\hline $\mathrm{O} 7^{\mathrm{i}}-\mathrm{Al} 4-\mathrm{O}^{\mathrm{vi}}$ & $108.60(6)$ \\
\hline $\mathrm{O} 4^{\mathrm{vi}}-\mathrm{A} 14-\mathrm{O} 2^{\mathrm{vi}}$ & $111.66(11)$ \\
\hline $\mathrm{O} 7^{\mathrm{i}}-\mathrm{A} 14-\mathrm{O} 2^{\mathrm{vi}}$ & $109.19(12)$ \\
\hline $\mathrm{O} 7^{\mathrm{vi}}-\mathrm{A} 14-\mathrm{O} 2^{\mathrm{vi}}$ & $108.46(10)$ \\
\hline $\mathrm{O} 4^{\mathrm{vi}}-\mathrm{A} 15-\mathrm{O} 3^{\mathrm{vii}}$ & $107.59(8)$ \\
\hline $\mathrm{O} 4^{\mathrm{vi}}-\mathrm{A} 15-\mathrm{O}^{\mathrm{xi}}$ & $111.47(7)$ \\
\hline $\mathrm{O}^{\mathrm{vii}}-\mathrm{A} 15-\mathrm{O}^{\mathrm{xi}}$ & $115.93(7)$ \\
\hline $\mathrm{O} 4^{\mathrm{vi}}-\mathrm{A} 15-\mathrm{O} 1^{\mathrm{i}}$ & $102.91(8)$ \\
\hline 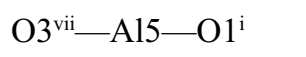 & $109.03(7)$ \\
\hline $\mathrm{O} 6^{\mathrm{xi}}-\mathrm{A} 15-\mathrm{O} 1^{\mathrm{i}}$ & $109.09(7)$ \\
\hline $\mathrm{A} 15^{\mathrm{xxii}}-\mathrm{O} 1-\mathrm{A} 15^{\mathrm{xxiv}}$ & $114.01(10)$ \\
\hline 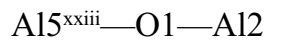 & $122.05(6)$ \\
\hline $\mathrm{Al} 5^{\mathrm{xxiv}}-\mathrm{O} 1-\mathrm{Al} 2$ & $122.05(6)$ \\
\hline $\mathrm{A} 15^{\mathrm{xxiii}}-\mathrm{O} 1-\mathrm{Pb} 2$ & $93.98(7)$ \\
\hline $\mathrm{A} 15^{\mathrm{xxiv}}-\mathrm{O} 1-\mathrm{Pb} 2$ & $93.98(7)$ \\
\hline $\mathrm{A} 12-\mathrm{O} 1-\mathrm{Pb} 2$ & $95.65(7)$ \\
\hline $\mathrm{A} 14^{\mathrm{ix}}-\mathrm{O} 2-\mathrm{A} 14^{\text {viii }}$ & $155.98(17)$ \\
\hline $\mathrm{A} 14^{\mathrm{ix}}-\mathrm{O} 2-\mathrm{Pb} 1$ & $101.69(8)$ \\
\hline $\mathrm{A} 14^{\mathrm{viii}}-\mathrm{O} 2-\mathrm{Pb} 1$ & $101.69(8)$ \\
\hline $\mathrm{A} 14^{\mathrm{ix}}-\mathrm{O} 2-\mathrm{Pb} 1^{\mathrm{xxiii}}$ & $86.97(9)$ \\
\hline 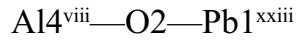 & $86.97(9)$ \\
\hline $\mathrm{Pb} 1-\mathrm{O} 2-\mathrm{Pb} 1^{\mathrm{xxiii}}$ & $117.87(11)$ \\
\hline $\mathrm{A} 14^{\mathrm{ix}}-\mathrm{O} 2-\mathrm{Pb} 2$ & $81.53(10)$ \\
\hline $\mathrm{A} 14^{\mathrm{vii}}-\mathrm{O} 2-\mathrm{Pb} 2$ & $81.53(10)$ \\
\hline $\mathrm{Pb} 1-\mathrm{O} 2-\mathrm{Pb} 2$ & $121.94(10)$ \\
\hline $\mathrm{Pb} 1^{\mathrm{xxiii}}-\mathrm{O} 2-\mathrm{Pb} 2$ & $120.19(8)$ \\
\hline $\mathrm{A} 15^{\mathrm{xxv}}-\mathrm{O} 3-\mathrm{Al} 3^{\mathrm{xiv}}$ & $125.65(8)$ \\
\hline $\mathrm{A} 15^{\mathrm{xxv}}-\mathrm{O} 3-\mathrm{A} 11^{\mathrm{xix}}$ & $119.80(7)$ \\
\hline $\mathrm{Al} 3^{\mathrm{xiv}}-\mathrm{O} 3-\mathrm{A} 11^{\mathrm{xix}}$ & $95.46(6)$ \\
\hline $\mathrm{A} 15^{\mathrm{xxv}}-\mathrm{O} 3-\mathrm{Pb} 1^{\mathrm{xxvi}}$ & $111.13(7)$ \\
\hline $\mathrm{Al} 3^{\mathrm{xiv}}-\mathrm{O} 3-\mathrm{Pb} 1^{\mathrm{xxvi}}$ & $97.22(5)$ \\
\hline
\end{tabular}




\begin{tabular}{|c|c|}
\hline $\mathrm{O} 7^{\mathrm{vi}}-\mathrm{Pb} 2-\mathrm{O} 4^{\mathrm{vii}}$ & $107.69(4)$ \\
\hline $\mathrm{O} 7^{\mathrm{vii}}-\mathrm{Pb} 2-\mathrm{O} 4^{\mathrm{vii}}$ & $58.03(4)$ \\
\hline $\mathrm{O} 4^{\text {viii_- }} \mathrm{Pb} 2-\mathrm{O} 4^{\text {vii }}$ & $174.49(4)$ \\
\hline $\mathrm{O} 4^{\mathrm{ix}}-\mathrm{Pb} 2-\mathrm{O} 4^{\text {vii }}$ & $115.37(5)$ \\
\hline $\mathrm{O} 6^{\mathrm{x}}-\mathrm{Pb} 2-\mathrm{O} 4^{\mathrm{vii}}$ & $57.61(4)$ \\
\hline $\mathrm{O}^{\mathrm{xi}}-\mathrm{Pb} 2-\mathrm{O} 4^{\mathrm{vii}}$ & $86.18(4)$ \\
\hline $\mathrm{O} 1-\mathrm{Pb} 2-\mathrm{O} 4^{\mathrm{vi}}$ & $116.66(5)$ \\
\hline $\mathrm{O} 7^{\mathrm{vi}}-\mathrm{Pb} 2-\mathrm{O}^{\mathrm{vi}}$ & 58.03 \\
\hline $\mathrm{O} 7^{\mathrm{vii}}-\mathrm{Pb} 2-\mathrm{O} 4^{\mathrm{vi}}$ & $107.69(4)$ \\
\hline $\mathrm{O} 4^{\mathrm{viii}}-\mathrm{Pb} 2-\mathrm{O} 4^{\mathrm{vi}}$ & $115.37(5)$ \\
\hline $\mathrm{O} 4^{\mathrm{ix}}-\mathrm{Pb} 2-\mathrm{O} 4^{\mathrm{vi}}$ & $174.49(4)$ \\
\hline $\mathrm{O} 6^{\mathrm{x}}-\mathrm{Pb} 2-\mathrm{O} 4^{\mathrm{vi}}$ & $86.18(4)$ \\
\hline $\mathrm{O} 6^{\mathrm{xi}}-\mathrm{Pb} 2-\mathrm{O}^{\mathrm{vi}}$ & $57.61(4)$ \\
\hline 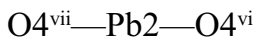 & $59.39(5)$ \\
\hline $\mathrm{O} 1-\mathrm{Pb} 2-\mathrm{O} 2$ & $146.03(6)$ \\
\hline $\mathrm{O} 7^{\mathrm{vi}}-\mathrm{Pb} 2-\mathrm{O} 2$ & $58.80(4)$ \\
\hline $\mathrm{O} 7^{\mathrm{vii}}-\mathrm{Pb} 2-\mathrm{O} 2$ & $58.80(4)$ \\
\hline 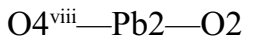 & $89.41(5)$ \\
\hline $\mathrm{O} 4^{\mathrm{ix}}-\mathrm{Pb} 2-\mathrm{O} 2$ & $89.41(5)$ \\
\hline $\mathrm{O} 6^{\mathrm{x}}-\mathrm{Pb} 2-\mathrm{O} 2$ & $145.70(4)$ \\
\hline $\mathrm{O}^{\mathrm{xi}}-\mathrm{Pb} 2-\mathrm{O} 2$ & $145.70(4)$ \\
\hline $\mathrm{O} 4^{\mathrm{vii}}-\mathrm{Pb} 2-\mathrm{O} 2$ & $92.50(5)$ \\
\hline 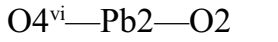 & $92.50(5)$ \\
\hline $\mathrm{O}^{\mathrm{xii}}-\mathrm{A} 11-\mathrm{O}^{\mathrm{vi}}$ & 180.0 \\
\hline $\mathrm{O} 5^{\mathrm{xii}}-\mathrm{A} 11-\mathrm{O} 3^{\mathrm{xiii}}$ & $95.60(6)$ \\
\hline $\mathrm{O} 5^{\mathrm{vi}}-\mathrm{A} 11-\mathrm{O} 3^{\mathrm{xii}}$ & $84.40(6)$ \\
\hline $\mathrm{O} 5^{\mathrm{xii}}-\mathrm{A} 11-\mathrm{O}^{\mathrm{xiv}}$ & $84.40(6)$ \\
\hline $\mathrm{O} 5^{\mathrm{vi}}-\mathrm{Al} 1-\mathrm{O} 3^{\mathrm{xiv}}$ & $95.60(6)$ \\
\hline $\mathrm{O} 3^{\mathrm{xii}}-\mathrm{A} 11-\mathrm{O} 3^{\mathrm{xiv}}$ & 180.0 \\
\hline $\mathrm{O} 5^{\mathrm{xii}}-\mathrm{A} 11-\mathrm{O} 3^{\mathrm{vii}}$ & $84.40(6)$ \\
\hline $\mathrm{O}^{\mathrm{vi}}-\mathrm{A} 11-\mathrm{O} 3^{\mathrm{vii}}$ & $95.60(6)$ \\
\hline $\mathrm{O} 3^{\mathrm{xii}}-\mathrm{A} 11-\mathrm{O} 3^{\mathrm{vii}}$ & $91.84(8)$ \\
\hline $\mathrm{O} 3^{\mathrm{xiv}}-\mathrm{A} 11-\mathrm{O} 3^{\mathrm{vii}}$ & $88.16(8)$ \\
\hline $\mathrm{O} 5^{\mathrm{xii}}-\mathrm{A} 11-\mathrm{O} 3^{\mathrm{xv}}$ & $95.60(6)$ \\
\hline $\mathrm{O}^{\mathrm{vi}}-\mathrm{A} 11-\mathrm{O}^{\mathrm{xv}}$ & $84.40(6)$ \\
\hline $\mathrm{O} 3^{\mathrm{xii}}-\mathrm{A} 11-\mathrm{O} 3^{\mathrm{xv}}$ & $88.16(8)$ \\
\hline $\mathrm{O} 3^{\mathrm{xiv}}-\mathrm{A} 11-\mathrm{O} 3^{\mathrm{xv}}$ & $91.84(8)$ \\
\hline $\mathrm{O} 3^{\mathrm{vii}}-\mathrm{A} 11-\mathrm{O} 3^{\mathrm{xv}}$ & 180.0 \\
\hline $\mathrm{O} 6^{\mathrm{xi}}-\mathrm{A} 12-\mathrm{O} 6^{\mathrm{xvi}}$ & 180.0 \\
\hline $\mathrm{O} 6^{\mathrm{xi}}-\mathrm{A} 12-\mathrm{O}^{\mathrm{xvii}}$ & $86.54(8)$ \\
\hline $\mathrm{O} 6^{\mathrm{xvi}}-\mathrm{A} 12-\mathrm{O}^{\mathrm{xvii}}$ & $93.46(8)$ \\
\hline $\mathrm{O} 6^{\mathrm{xi}}-\mathrm{A} 12-\mathrm{O}^{\mathrm{x}}$ & $93.46(8)$ \\
\hline $\mathrm{O} 6^{\mathrm{xvi}}-\mathrm{A} 12-\mathrm{O}^{\mathrm{x}}$ & $86.54(8)$ \\
\hline
\end{tabular}

\begin{tabular}{|c|c|}
\hline $\mathrm{A} 11^{\mathrm{xix}}-\mathrm{O} 3-\mathrm{Pb} 1^{\mathrm{xxvi}}$ & $103.48(5)$ \\
\hline $\mathrm{A} 15^{\mathrm{xxy}}-\mathrm{O} 3-\mathrm{Sr} 1^{\mathrm{xxvi}}$ & $111.13(7)$ \\
\hline $\mathrm{A} 13^{\mathrm{xiv}}-\mathrm{O} 3-\mathrm{Sr} 1^{\mathrm{xxvi}}$ & $97.22(5)$ \\
\hline $\mathrm{A} 11^{\mathrm{xix}}-\mathrm{O} 3-\mathrm{Sr} 1^{\mathrm{xxvi}}$ & $103.48(5)$ \\
\hline $\mathrm{Al} 5^{\mathrm{viii}}-\mathrm{O} 4-\mathrm{A} 14^{\mathrm{viii}}$ & $139.38(10)$ \\
\hline $\mathrm{Al} 5^{\mathrm{viii}}-\mathrm{O} 4-\mathrm{Pb} 2^{\mathrm{vi}}$ & $92.13(6)$ \\
\hline $\mathrm{A} 14^{\mathrm{viii}}-\mathrm{O} 4-\mathrm{Pb} 2^{\mathrm{vi}}$ & $125.63(8)$ \\
\hline $\mathrm{A} 15^{\mathrm{viii}}-\mathrm{O} 4-\mathrm{Sr} 2^{\mathrm{vi}}$ & $92.13(6)$ \\
\hline $\mathrm{A} 14^{\mathrm{viii}}-\mathrm{O} 4-\mathrm{Sr} 2^{\mathrm{vi}}$ & $125.63(8)$ \\
\hline $\mathrm{A} 15^{\text {viii }}-\mathrm{O} 4-\mathrm{Pb} 2^{\text {viii }}$ & $88.60(6)$ \\
\hline 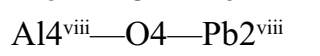 & $87.68(6)$ \\
\hline $\mathrm{Pb} 2^{\mathrm{vi}}-\mathrm{O} 4-\mathrm{Pb} 2^{\text {viii }}$ & $115.37(5)$ \\
\hline $\mathrm{Sr} 2^{\mathrm{vi}}-\mathrm{O} 4-\mathrm{Pb} 2^{\mathrm{viii}}$ & $115.37(5)$ \\
\hline 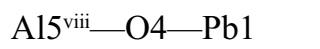 & $84.88(6)$ \\
\hline $\mathrm{A} 14^{\mathrm{vii}-\mathrm{O} 4-\mathrm{Pb} 1}$ & $80.74(6)$ \\
\hline $\mathrm{Pb} 2^{\mathrm{vi}}-\mathrm{O} 4-\mathrm{Pb} 1$ & $90.68(5)$ \\
\hline $\mathrm{Sr} 2^{\mathrm{vi}}-\mathrm{O} 4-\mathrm{Pb} 1$ & $90.68(5)$ \\
\hline 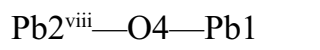 & $153.38(5)$ \\
\hline $\mathrm{A} 11^{\mathrm{viii}}-\mathrm{O} 5-\mathrm{Al} 3^{\mathrm{xxvii}}$ & $96.02(7)$ \\
\hline $\mathrm{A} 11^{\mathrm{viii}}-\mathrm{O} 5-\mathrm{Al} 3^{\text {viii }}$ & $96.02(7)$ \\
\hline $\mathrm{A} 13^{\mathrm{xxvii}}-\mathrm{O} 5-\mathrm{A} 13^{\mathrm{viii}}$ & $95.28(9)$ \\
\hline $\mathrm{A} 11^{\mathrm{viii}}-\mathrm{O} 5-\mathrm{Sr} 1^{\mathrm{xxiii}}$ & $156.90(10)$ \\
\hline $\mathrm{A} 13^{\mathrm{xxvii}}-\mathrm{O} 5-\mathrm{Sr} 1^{\mathrm{xxiii}}$ & $99.48(6)$ \\
\hline 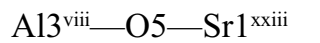 & $99.48(6)$ \\
\hline $\mathrm{A} 11^{\mathrm{viii}}-\mathrm{O} 5-\mathrm{Pb} 1^{\mathrm{xxiii}}$ & $156.90(10)$ \\
\hline $\mathrm{A} 13^{\mathrm{xxvii}}-\mathrm{O} 5-\mathrm{Pb} 1^{\mathrm{xxiii}}$ & $99.48(6)$ \\
\hline 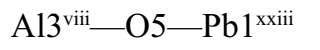 & $99.48(6)$ \\
\hline $\mathrm{A} 15^{\mathrm{xi}}-\mathrm{O} 6-\mathrm{A} 12^{\mathrm{xxviii}}$ & $127.55(7)$ \\
\hline $\mathrm{A} 15^{\mathrm{xi}}-\mathrm{O} 6-\mathrm{A} 13^{\mathrm{xxix}}$ & $119.36(7)$ \\
\hline $\mathrm{A} 12^{\mathrm{xxviii}}-\mathrm{O} 6-\mathrm{A} 13^{\mathrm{xxix}}$ & $94.41(6)$ \\
\hline $\mathrm{Al} 5^{\mathrm{xi}}-\mathrm{O} 6-\mathrm{Pb} 2^{\mathrm{xi}}$ & $94.48(6)$ \\
\hline $\mathrm{Al} 2^{\mathrm{xxviii}}-\mathrm{O} 6-\mathrm{Pb} 2^{\mathrm{xi}}$ & $89.02(5)$ \\
\hline $\mathrm{A} 13^{\mathrm{xxix}}-\mathrm{O} 6-\mathrm{Pb} 2^{\mathrm{xi}}$ & $132.22(6)$ \\
\hline $\mathrm{Al} 4^{\mathrm{xxiii}}-\mathrm{O} 7-\mathrm{Al}^{\mathrm{viii}}$ & $118.72(9)$ \\
\hline $\mathrm{A} 14^{\mathrm{xxiii}}-\mathrm{O} 7-\mathrm{Pb} 2^{\text {viii }}$ & $100.62(7)$ \\
\hline $\mathrm{Al} 4^{\text {viii }-O} \mathrm{O} 7-\mathrm{Pb} 2^{\text {viii }}$ & $105.19(7)$ \\
\hline $\mathrm{Al} 4^{\text {xxiii }}-\mathrm{O} 7-\mathrm{Sr} 2^{\text {viii }}$ & $100.62(7)$ \\
\hline $\mathrm{Al} 4^{\mathrm{viii}}-\mathrm{O} 7-\mathrm{Sr} 2^{\mathrm{viii}}$ & $105.19(7)$ \\
\hline $\mathrm{A} 14^{\mathrm{xxiii}}-\mathrm{O} 7-\mathrm{Pb} 1^{\mathrm{xxiii}}$ & $129.96(8)$ \\
\hline 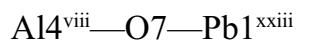 & $95.99(7)$ \\
\hline $\mathrm{Pb} 2^{\text {viii }-O} 7-\mathrm{Pb} 1^{\mathrm{xxiii}}$ & $103.69(5)$ \\
\hline $\mathrm{Sr} 2^{\text {viii }}-\mathrm{O} 7-\mathrm{Pb} 1^{\text {xiii }}$ & $103.69(5)$ \\
\hline
\end{tabular}

$111.13(7)$

$97.22(5)$

$103.48(5)$

$139.38(10)$

$92.13(6)$

$125.63(8)$

$92.13(6)$

$125.63(8)$

$88.60(6)$

$87.68(6)$

$115.37(5)$

$84.88(6)$

$80.74(6)$

$90.68(5)$

$90.68(5)$

$153.38(5)$

$96.02(7)$

$96.02(7)$

$156.90(10)$

$99.48(6)$

$99.48(6)$

$156.90(10)$

$99.48(6)$

$127.55(7)$

$119.36(7)$

$94.41(6)$

$94.48(6)$

$132.22(6)$

$118.72(9)$

$100.62(7)$

$05.19(7)$

$100.62(7)$

$105.19(7)$

$129.96(8)$

$95.99(7)$

$103.69(5)$

Symmetry codes: (i) $x, y-1, z$; (ii) $x, y,-z+1$; (iii) $x, y, z-1$; (iv) $x, y-1,-z$; (v) $x, y,-z$; (vi) $-x+1 / 2, y-1 / 2,-z+1 / 2$; (vii) $-x+1 / 2, y-1 / 2, z-1 / 2$; (viii) $-x+1 / 2, y+1 / 2,-z+1 / 2$; (ix) $-x+1 / 2, y+1 / 2, z-1 / 2$; (x) $-x+1,-y+1, z-1$; (xi) $-x+1,-y+1,-z+1$; (xii) $x-1 / 2,-y+1 / 2, z+1 / 2 ;$ (xiii) $x-1 / 2,-y+1 / 2, z-1 / 2$; (xiv) $-x+1 / 2, y-1 / 2,-z+3 / 2$; (xv) $x-1 / 2,-y+1 / 2,-z+3 / 2$; (xvi) $x-1, y, z-1$; (xvii) $x-1, y,-z+1$; (xviii) $-x,-y+1,-z$; (xix) $-x+1 / 2, y+1 / 2,-z+3 / 2 ;$ (xx) $x-1 / 2,-y+3 / 2, z+1 / 2$; (xxi) $-x+1,-y+1, z$; (xxii) $x-1, y, z$; (xxiii) $x, y+1, z$; (xxiv) $x, y+1,-z$; (xxv) $-x+1 / 2, y+1 / 2, z+1 / 2$; (xxvi) $x, y, z+1$; (xxvii) $x+1 / 2$, $-y+3 / 2, z-1 / 2$; (xxviii) $x+1, y, z+1$; (xxix) $x+1, y, z$. 